\title{
The Potential of A "Virtual" Construction Site for Automation Planning and Analysis
}

\author{
Chris Hendrickson and Daniel R. Rehak
}

Department of Civil Engineering, Camegie Mellon University, Pittsburgh, PA 15213-3890 USA

Internet: cth@cmu.edu, rehak@cmu.edu,FAX: 412-268-7813

\begin{abstract}
Existing simulation models for construction operations typically rely on geometric objects moving through an abstract space or on monte carlo simulation models without specific geometric representations. Physicallybased models of such operations coupled with new technologies for user interaction offer tremendous potential for improving the realism and usefulness of simulation models. This paper describes the potential of a "virtual" construction site for automation planning and analysis.
\end{abstract}

\section{Introduction}

Concepts for automation and use of robotic equipment on construction sites are evolving to encompass integrated equipment systems and sophisticated, distributed processing architectures. The evolution of construction robotics concepts can best be traced in the ten proceedings from the International Symposium on Automation and Robotics in Construction. Initial concepts for construction robotics focused on singlepurpose machines based on industrial robot arm configurations. Geometry-based CAD systems were used in early systems for site modeling and planning. Automation schemes were often based upon industrialization of component fabrication [Warszawski 90], or trains of numerical controlled equipment used in repetitive work such as roadway paving [Skibniewski 90]. More recent work in automation design has emphasized changes in process design (to allow more effective automation) and overall integrated systems for tasks such as building assembly. Distributed sensing systems and general site models, such as ConSolve's SitePlanner, are also beginning to appear, particularly in response to regulatory requirements on environmental remediation sites.

While more complex automation systems are being developed, there are no comprehensive systematic methods available to assess alternatives for site automation. Prior work has relied on theoretical studies, simple geometric models, or physical experimentation.

A computer-based, "virtual construction site" (VCS) model could be used to investigate alternative designs for sensing, site modeling, distributed processing and automation on construction sites. The virtual construction site model enables a much more realistic assessment of automation and process design altematives and strategies than is available with existing, geometry-based computer-aided design (CAD) models.

Existing CAD systems, e.g., Bechtel's Walk-thru and AutoCAD have powerful capabilities for geometric 
representation, visualization and animation of three-dimensional models, but they do not integrate genuine physical models of motion, gravity, fluid flows or other physical phenomena. For example, a typical CAD model would not recognize that a load swaying from a crane has inertia or that a piece of equipment might overtum and fall under the influence of gravity. Considering realistic issues in automation planning, such as sensor placement and perception range or altematives for automated system control, requires moving beyond only the considerations of the simple geometric interferences supported by existing CAD systems.

With a realistic model of construction sites, encompassing open user actions and physical phenomena along with geometric representations, we can assess different schemes for site automation more rapidly and cost effectively than would be possible in field trials or physical laboratory simulations. For example, we might wish to evaluate the effectiveness of multiple range sensors placed on site for the purpose of supporting the activity of a robotic excavator. Each range sensor would have a particular viewpoint and capability. The sensor model incorporated into the VCS would have limited access to the description (both geometric and operational state) of the site, based on the sensor capabilities, and data from the VCS model would be fitered to reflect the errors inherent in the sensor operation. As another example, we might evaluate different heavy lift strategies with respect to the possibility of load shifting and allowable cable strains. Incorporating models of physical phenomena in a VCS would allow engineering analysis of gravity and forces on site components.

Development of the VCS model alone may be beneficial for many purposes. In a sense, the VCS would represent an example of the next generation of CAD systems in which engineering information could be accurately simulated. Rather than requiring engineers and designers to explicitly select and model those aspects which are considered, a virtual CAD system for a particular domain would automatically incorporate considerations of applicable phenomena.

The term virtual deserves explanation. We use the term in the same form as is traditional in mechanics or computations (e.g., virtual work, virtual memory), an imaginary system which mimics the behavior of the real counterpart which it models. In addition, our vision includes making the user (e.g., site planner, equipment operator) a part of the virtual world, able to directly interact with it. The VCS will be a "virtual reality" world. Connected to typical "virtual reality" interaction devices such as display goggles or instrumented data gloves, the VCS would permit direct interaction with the environment, such as fluid flows or structural strains. For example, the movement of groundwater components in response to different pumping strategies might be represented by different colors, or "felt" through instrumented flow meters. This type of model might be useful in the design and selection of hazardous site remediation strategies. Or the VCS model could be adopted for site logistics planning, in which the planner could "move" equipment and laydown areas, and the feedback would reflect cost or time implications of the selection.

This paper reviews existing work relevant to the development of a virtual construction site, defines system requirements, and considers the overall feasibility and desirability of a VCS system. We begin with an example of a crane pick-and-place operation.

\section{An Example of Physically-Based Simulation Modeling: Crane Operations}

Suppose that a crane is being used to place beams in a structure. The crane is at a fixed location on the site. A typical pick-and-place operation involves several steps:

1. identify the beam to be placed from those in the laydown area. 
2. swing and boom the crane over the beam.

3. drop the hooks to the beam.

4. attach the hooks to the beam.

5. lift, swing and boom the beam into place.

6. position the beam into the final placement position.

7. temporarily attach the beam to the structure.

8. unhook the beam from the crane.

9. move the crane for the next operation.

In a simple model which might be animated in a state-of-the-art computer-aided design software package, the crane hooks and the beam move in a smooth path, from the initial crane location, to the pick, through the lift and to the final position. In the real situation, there are a number of considerations which complicate the operation:

- Beam physics. The beam is a three-dimensional object, which has mass and stiffness. This implies that the inertia of the beam will influence the path of the crane, and the beam as a load will swing from the crane. Likewise, the beam will deflect when lifted, and both the beam's three dimensional shape and stiffness may influence where the hooks can be attached, and the deflection will influence the final placement of the beam in the structure.

- Crane physics. The crane also has mass and stiffness. The crane body, boom, cables and hooks and grappels all are influenced by their relative strength and mass. For example, when unloaded, the hook will drag behind the path of the tip of the boom, and the hooks will swing like pendula. With the load attached, the cables will elongate, the boom will deflect (due to the load and the joint compliance), and the load will again behave with a pendula-like motion.

- Operator Action. The actual crane and beam motion will be driven by the operator actions in swinging and lifting the load. The speed of the engine and velocity of the elements of the crane and timing of the operator actions will drive the system.

- Environment. The crane and the load are not uncoupled from the world in which they are operating. Simple factors, such as wind (varying dynamically during the lift) combined with the mass and aerodynamics of the elements combine to influence the actual motions. Other influence, such as temperature also may be important (picking the beam from a cold shaded area and placing it into a sunny area where the thermal effect on beam length influences fit).

All of these physical constraints will combine to determine the actual path of the crane. A more realistic model of behavior will incorporate these effects:

1. assume the crane is initially at rest in some configuration (influenced by the self-weight and stiffness of the crane). 
2. identify the beam to be placed.

3. swing and boom the crane towards the load. In the process, incorporate the mass of the crane, boom, cables, hooks, etc., computing the path of the boom tip and hooks, as driven by the operator's action and the environment.

4. drop the hooks to the beam (this and all other actions are influenced by the physics, etc., describe above).

5. attach the hooks to the beam.

6. lift, swing and boom the beam into place.

7. position the beam into the final placement position.

8. temporarily attach the beam to the structure.

9. unhook the beam from the crane.

10. move the crane for the next operation.

The above sequence just describes the basic mechanics of the pick and place operation. In addition, the processes of overall control and communications between the different people involved in the process may involve significant management or process design issues and thus deserve attention in the model. For example, the crane operator might not be able to see the hooks and load at various times in the operation (e.g., the beam is picked from behind another building or pile of materials, or the placement position is obscured from the operator's view). Thus a realistic model should also incorporate additional factors:

- Project World. The crane operates in a three-dimensional world of the entire construction site. This world is dynamically changing, with some elements changing slowly over days, such as the site and the structure being built, and other moving quickly, such as other cranes.

- Communications. The process of inter-person or electronic communication may involve delays or loss of information.

- Control. Control at a simple level is no more than specifying which element is to be placed and its final location. More detailed operational control consists of planning the path of the crane, or optimizing the power of the crane engine to the hoist and hydraulics to minimize fuel consumption.

- Sensing. Automated control of various aspects of the process or even direct monitoring of the operations will involve sensors that may have limited range. delays or other constraints.

Incorporation of these various physical constraints and control issues may be crucial for developing an accurate portrayal of crane movements and other construction operations. Similar sets of constraints may be developed for other construction operations, including issues such as soil or groundwater movements.

While developing and validating physical models of the type described above may be time-consuming, the basic models should be able to be re-used in numerous construction applications in the same way that standard component representations are re-used in CAD models. Moreover, simulation of automation and control options in a simulation model may be much less expensive and faster than actual field trials. From this example of possibilities, we turn to examine the existing state of the art. 


\section{Virtual Reality}

In most conventional computer applications, the computer and the user are two different participants in an environment with a limited and predefined set of interactions. The actions which the computational agent can perform are planned before the system is implemented, as are the types of output it.will produce. The user has a restricted communications channel to the machine, typically just a keyboard and display. Unacceptable and unplanned inputs are ignored, or worse, cause failures.

"Virtual reality" attempts to fully integrate the user with the computer system, bringing the user into the environment of a single modeled world. In the extreme, all of the user's actions are monitored (e.g., he is in a fully instrumented body suit on a sensing platform). All the user's sensory input is provided by the machine, typically a head-mounted display that presents images and blocks the rest of the visual field. Aural feedback is also controlled, along with forces and motions. The actions which the computer performs are determined by the user actions, and all of the information which the user relies on to control his actions is provided by the machine.

While no virtual reality systems exist which completely bring the user into the computer's environment, applications and developments in place are moving towards a tighter coupling of the user and computer. Current applications model a number of phenomena in which the actions dynamically evolve throughout a simulation. One of the most advanced applications is BattleTech, a "video game" environment in which each member of a group of players individually controls a robotic "tank" in a battle situation over a large terrain [Grimes 92]. In BattleTech, the computer provides the artificial environment (weather, terrain), moves the tanks through the world, and mediates the interactions among the players. Developments are underway in other areas such as entertainment, architecture, and medical and scientific simulations [Grimes 91 . Haggerty 91$]$.

One common virtual world is a geometric model in which the user can navigate. Using sensors, the computer interprets user actions in terms of walking and looking in different directions in the world. As the user moves though the space, the display is automatically changed to reflect location and view direction. Such applications require only geometric models of the world, and a simple model of user motion. A head-mounted display or stereo display presents the scene to the user, and an instrumented glove senses the movement and position of the fingers and hand. Different hand gestures indicate movement, speed, and view direction.

Simple virtual worlds only model geometry and visual effects (e.g., lighting), and user interaction is limited to moving in the world. Letting the user modify the world, by moving objects, opening doors, etc., provides the next level of complexity. The behavior and physics of objects and the world are introduced in more complex environments. Displays can range from simple wire-frame models to realistic images, according to the requirements of the domain. Head-mounted displays which block all other vision provide the most realistic interaction, however a single or stereo monitor may be sufficient in some applications. User interaction can be simulated with common devices such as mice or joysticks, while most simulations use three-dimensional input devices such as wands, data gloves, and in the extreme, body suits. Most of the complexity in building a virtual world is in building the models, programming the displays, and controlling user interaction. 


\section{Virtual Reality Applications in Construction}

No virtual reality applications have yet appeared for construction automation and planning, although researchers are beginning to discuss the idea [Kangari 92]. A number of developments over the last two decades lead towards virtual reality applications in the AEC community. While most of these do not qualify as true virtual reality systems, they all share characteristics with current virtual reality work.

One of the first attempts to build a virtual world for visualization dates from the early 1970s, when as part of the plan to locate the I. M. Pei H. E. Johnson Museum of Art on the Cornell University campus, a walk-though was generated. This consisted of a film sequence of travel throughout the campus, overlaid with a computer-generated display of the facility, permitting different siting options to be explored. While not interactive, it illustrates the same "virtual" concept: the building displays show realistic views of the new structure at the potential sites.

This idea led directly to similar developments, either combining film and computer images, or relying totally on a computer generated environment. A computer-generated display can be combined with video from a video disk. Random access of the video disk lets the user select the viewing sequence, and the display can be generated to properly overlay on the video. More realistic worlds require the computer to display the entire model, and also permit the viewer to chose his path and sight line, rather than following a predefined path.

Such walk-through (or fly-through) animations have been used on many architectural projects. Current systems extend the visualizations to include high-quality renderings of surfaces, lighting, etc. While many applications require artificial means to specify the path (e.g., trace a path on a 2-D plan view), recent systems incorporate head-mounted displays and data gloves.

The state of the art in architectural visualization is represented by the work of Brooks et al.. at UNC Chapel Hill, and in AEC, by the Walk-thru program developed by Bechtel. These systems have been applied primarily to view altemative designs. In a similar application, Stone \& Webster has used a walkthrough system to plan placement of large equipment in a power plant; the equipment follows the path, and interference detections are used to eliminate infeasible moves. Such a system goes beyond simple display and includes interactions of parts of the world. Fully interactive systems of this type can simplify complex planning.

Simple animation systems for viewing construction operations have been in place for many years (e.g., [Kano 84] or [Stouffs 93]). These systems illustrate a construction sequence, displaying an evolving geometric model, following an erection sequence derived from the schedule.

The first commercial AEC virtual reality application is the Virtual Showroom, a kitchen planning system developed by Matsushita Electric Works. A three-dimensional model of the kitchen is built from the user's specifications, and by using a headset and data glove, the user can move around the space, "touch" counter-tops, open doors, etc. Physical models here include simple interference detection.

Most of the current AEC work in virtual reality is aimed towards visualization. Virtual worlds which include complex physical phenomena are beginning to appear, as are more realistic displays and more complex modes of user interaction. Virtual reality applications extend the earlier visualization, simulation and animation work by incorporating of all these features.

All of the basic virtual reality technologies needed to build a VCS are in place. Sufficient computing power is available, and significant work is underway in visualization and developing physical models. Details 
of these evolutions have been presented in the various SIGGRAPH proceedings. The ISARC proceedings have presented work such as force-cognizant excavation, crane collision avoidance systems, and models of various robotic agents. Together, sufficient information and tools are available to build a VCS.

\section{Impact of Physically-Based Virtual Reality for Construction}

While statements about the concepts of using virtual worlds in civil engineering have been made [Fenves 91], there has been little substantive effort towards developing a "virtual construction site system" or using a virtual environment for modeling and analysis in construction automation and planning. We believe that this next generation of construction simulation tools should incorporate both enhanced user interaction mechanisms common in "virtual reality" demonstrations and games, and a variety of physically-based models to greatly improve the realism and accuracy of such simulation tools.

At this time, the appropriate representations and software architecture for a "virtual construction site" have not yet been determined. Efforts to develop physically-based models in other domains typically rely on distributed processing and object-oriented representations. With proper attention, particular model types might be capable of being moved in a modular fashion from one application to another. We are already investigating this type of modularity in a crane model. However, the peculiarities of many construction operations and concerns suggest that systems developed for other domains will be either serious deficient or inappropriate for construction applications.

In sum, simulation models are a demonstrated way in which to improve the overall construction process. We anticipate new technologies in computers and communications also playing major roles in construction process improvements. Work on this general problem can help lead the construction industry into the next century.

\section{References}

[Fenves 1991] Fenves, S. J., "The Potentials of Computer-Based Technologies in Civil Engineering," Twenty-Ninth Henry M. Shaw Lecture in Civil Engineering, Department of Civil Engineering, North Carolina State University, Raleigh, NC, December, 1991.

[Kano 1984] Kano, N., and Tamura, Y., "Application of Computer Graphics in Construction Planning and Scheduling," Proceedings Workshop Conference on Robotics in Construction, Pittsburgh, PA, Department of Civil Engineering, Carnegie Mellon University, June 17-20 1984.

[Grimes 1991] Grimes, J., "Virtual Reality 91 Anticipates Future Reality," IEEE Computer Graphics \& Applications, Vol. 11, No. 6, pp. 81-83, November 1991.

[Grimes 1992] Grimes, J., "Virtual Reality Goes Commercial with a Blast,"IEEE Computer Graphics \& Applications, Vol. 12, No. 2, pp. 16-17, March 1992. 
[Haggerty 1991] Haggerty, M., "Virtual Reality Dominates SIGGRAPH," IEEE Computer Graphics \& Applications, Vol. 11, No. 5, pp. 14-15, September 1991.

[Kangari 1992] Kangari, R., and Akimoto, M., "Artificial Reality in Robotics and A/E/C Firms," Proceedings 9 th International Symposium on Automation and Robotics in Construction, Tokyo, June 1992.

[Skibniewski 1990] Skibniewski, M., and Hendrickson, C., "Automation and Robotics for Road Construction and Maintenance," ASCE Journal of Transportation Engineering, American Society of Civil Engineers (ASCE), Vol. 116, No. 3, pp. 261-272, May/June 1990.

[Stouffs 1993] Stouffs, R., Krishnamurti, R., Lee, S., and Oppenheim, I., "Construction Process Simulation with Rule-Based Robot Path Planning," Proceedings 10th International Symposium on Automation and Robotics in Construction, Houston, Texas, May 1993.

[Warszawski 1990] Warszawski, A., Industrialization and Robotics in Building, Harper and Row, 1990. 